\title{
Measuring B-Type Natriuretic Peptide From Capillary Blood or Venous Sample: Is It the Same?
}

\author{
Renato De Vecchis ${ }^{\mathrm{a}, \mathrm{b}}$, Carmelina Ariano ${ }^{\mathrm{a}}$
}

\begin{abstract}
Background: In recent years, several systems have been implemented to achieve quick and non-invasive measurements of B-type natriuretic peptide (BNP). Among them, Alere ${ }^{\mathrm{TM}}$ Heart Check (AHC) BNP test represents the most recent advancement. It is a rapid point-ofcare (POC) immunoassay, projected for measuring BNP directly from a capillary whole blood sample. This study aimed at comparing the analytical and clinical performances of this new POC to our reference method (Abbott Architect System).
\end{abstract}

Methods: One hundred eleven patients with stable chronic heart failure (CHF) referring to one cardiac rehabilitation center were enrolled from December 2013 to May 2015. These patients were subjected to a simultaneous capillary (AHC) and plasma (Abbott) BNP measurements. Clinical and analytical performances of AHC were assessed and compared to the reference method.

Results: Capillary BNP showed a good correlation with the reference method $(\mathrm{r}=0.94, \mathrm{P}<0.0001)$, although the values diverged when BNP was higher than $1,500 \mathrm{pg} / \mathrm{mL}$. Indeed, the AHC had a relatively poor precision and the coefficient of variability was $10.1 \%$ and $18 \%$ for low and high controls, respectively. However, both methods showed similar diagnostic performances in discriminating the patients with heart failure in New York Heart Association (NYHA) class I from those belonging to NYHA classes II-III, with values of area under the curve (AUC) of 0.983 and 0.984 , respectively, and equivalent sensitivity, specificity, and positive and negative likelihood ratios.

Conclusion: The AHC BNP test is a good POC able to provide reliable information about the hemodynamic status of CHF patients, especially of those belonging to NYHA classes I-III.

Keywords: B-type natriuretic peptide; Point-of-care; Immunoassay; Heart failure; Capillary blood; Venous blood

Manuscript accepted for publication April 15, 2016

aCardiology Unit, Presidio Sanitario Intermedio "Elena d'Aosta", ASL Napoli 1 Centro, Napoli, Italy

${ }^{\mathrm{b}}$ Corresponding Author: Renato De Vecchis, Cardiology Unit, Presidio Sanitario Intermedio "Elena d'Aosta", ASL Napoli 1 Centro, via Cagnazzi 29, 80137 Napoli, Italy (Personal postal address: via P. Gaurico 21, 80125 Napoli, Italy).Email: devecchis.erre@virgilio.it

doi: http://dx.doi.org/10.14740/cr468e

\section{Introduction}

The B-type natriuretic peptide (BNP) and the amino-terminal fragment of the pro-B-type natriuretic peptide, known as NTproBNP, are currently seen as gold standard biomarkers for the diagnosis and prognostic stratification of heart failure, according to international guidelines $[1,2]$. These biomarkers are not only able to discriminate the origin of dyspnea (cardiac versus non-cardiac dyspnea, e.g. bronchial asthma) in the setting of emergency medicine $[1,2]$, but they are also useful for better managing patients with stable chronic heart failure (CHF). Indeed, in the last decade, there have been developments in the concept of BNP-guided therapy, in which the serial measurements of BNP over time are used to assess the effectiveness of drug treatment for heart failure, and to guide possible adjustments and modifications to dosage. However, the current methods for the measurement of BNP are invasive, requiring a venous blood sample (e.g. from the antecubital vein of the arm), with specific pre-analytical requirements in order to make the interpretation of the results feasible. In this context, the relative discomfort resulting from venipuncture and the relatively long period of time needed to get the test results could be an obstacle to a strict careful monitoring of the patient with repeated tests for BNP from venous blood. These constraints prevent the monitoring of the BNP levels at home or at the office of the general practitioner and require that patients are directed to centers for laboratory investigations. To overcome these limitations, some systems suitable for allowing rapid and non-invasive measurement of multiple biological parameters, including the BNP [3], have been developed. Ideally, such a so-called "point-of-care" (POC) should measure the BNP from capillary blood, similarly to blood sugar measurement from the fingertip in diabetic patients. These POCs have many advantages, e.g., greater convenience and speed of detection of the results than in the reference methods. A system for detecting analytes at the bedside (POC) would enable easy and quick BNP monitoring even in a remote way, and would be useful for routine checks at the offices of the primary care physician or of the community cardiologist. In addition, POC may also be used in hospitals for the rapid monitoring of BNP-guided treatment of heart failure during an outpatient visit in the Department of Cardiology and for a rapid triage of patients with acute dyspnea in the Emergency Department. However, although promising, the preparation and evaluation of a POC as a diagnostic rou- 
Table 1. Comparison of the Analytical Performance of POC* and Lab-Based ${ }^{\star *}$ BNP Assays [6, 8, 9]

\begin{tabular}{lllll}
\hline System & Sample type & CV low control & CV high control & Reference \\
\hline Abbott Architect** & EDTA plasma & $5.5 \%$ & $3.2 \%$ & {$[6]$} \\
Abbott i-STAT* & EDTA whole blood & $14 \%$ & $9.8 \%$ & {$[6]$} \\
Alere Heart Check* & Capillary whole blood & $10.1 \%$ & $18 \%$ & This study \\
& & $8.4 \%$ & $14.1 \%$ & {$[8]$} \\
Alere Triage* & EDTA plasma & $9.2 \%$ & $11.4 \%$ & {$[9]$} \\
\hline
\end{tabular}

CV: coefficient of variation; EDTA: ethylenediaminetetraacetic acid.

tine tool requires that this kind of method is conveniently assessed to test adequately its reliability in comparison with the reference methods, i.e. those used in laboratories for blood analyses. Until a short time ago, the two POCs available for the measurement of BNP were the Alere ${ }^{\mathrm{TM}}$ Triage [4] and the Abbott i-STAT $[5,6]$, the latter using venous or arterial whole blood, while the former uses plasma treated with ethylenediaminetetraacetic acid (EDTA) as a chelating agent (Table 1) [6-9]. In contrast, the Alere ${ }^{\mathrm{TM}}$ Heart Check BNP test is the first POC designed as a type of immunological assay for the measurement of BNP from whole untreated capillary blood. Two preview studies demonstrated the safety and feasibility of this test at home for patients [10] and healthcare providers [8]. However, no peer-reviewed studies have compared the Alere $^{\mathrm{TM}}$ Heart Check BNP test with the reference methods used in the medical laboratory, except for the data provided by the Alere ${ }^{\mathrm{TM}}$ Heart Check BNP test compared with the Beckman Coulter Access II BNP assay [9]. Therefore, the aim of this study was to evaluate the clinical performance of this new POC to measure BNP by comparing it with our consolidated reference method (Abbott Architect System), as an analytical prerequisite for a large deployment of this system in the hospital.

\section{Methods}

\section{Patients}

We enrolled 111 patients with stable CHF who were followed at the two cardiac rehabilitation units that participated in our search (E.d'A. and S.M.d.P.) from February 2013 to January 2015. For enrollment, only patients with CHF in New York Heart Association (NYHA) classes I-III were considered, irrespective of the origin (coronary artery disease, cardiomyopathy, valvular disease, etc.) of their cardiac insufficiency. $\mathrm{Pa}$ - tients with a clinical picture of instability (i.e., patients with acute decompensated heart failure) were excluded. Both $\mathrm{CHF}$ patients with reduced left ventricular ejection fraction (LVEF) and those with preserved LVEF were included in the study. Heart failure was defined as the inability of the heart to pump sufficiently to maintain blood flow to meet the body's needs. For clinical categorization's purposes, we used the NYHA classes, according to the usual significance attributed to each class, as reported in Table 2.

\section{BNP measurements}

These patients were subjected to simultaneous capillary (Aler$\mathrm{e}^{\mathrm{TM}}$ Heart Check System) and plasma (Abbott Architect System) BNP measurement. Plasma was obtained from EDTA blood samples centrifuged at $3,500 \mathrm{~g}$ for $15 \mathrm{~min}$ at $4{ }^{\circ} \mathrm{C}$. Plasma BNP was subsequently measured on an Abbott Architect i2000. The Alere ${ }^{\mathrm{TM}}$ Heart Check BNP test used capillary whole blood to measure BNP. This method was a one-step immunoassay that uses biotinylated anti-BNP monoclonal antibody and streptavidin-coated magnetic solid-phase particles that were used to attract the immune binding of latex particles coupled with horseradish peroxidase and a monoclonal antibody fragment. This generated an electrochemical detection signal proportional to the level of BNP in the patient sample. The limits of detection were $10-4,955 \mathrm{pg} / \mathrm{mL}$. The Alere ${ }^{\mathrm{TM}}$ Heart Check was ready to use on a static or mobile device; capillary blood obtained by pricking was deposited on a single-use test strip, and could be performed by patients themselves. There was no pre-analytical requisite for this method. Results were provided within $15 \mathrm{~min}$. The Abbott Architect System was the lab-based system used in our hospitals for the routine measurement of plasma BNP; this method was valid and considered as a reference method. It was based on a two-step sandwich immunoassay using monoclonal antibodies specific for human BNP; detection was done by chemiluminescence. The limits

Table 2. NYHA Classification

\begin{tabular}{ll}
\hline NYHA class & Symptoms \\
\hline I & $\begin{array}{l}\text { Heart disease, but without resulting limitations of physical activity. Ordinary physical } \\
\text { activity is not limited, but symptoms appear for activities out of the ordinary. }\end{array}$ \\
II & $\begin{array}{l}\text { The patient is fine at rest but ordinary physical activity causes the appearance of symptoms. } \\
\text { III }\end{array}$ \\
IV & Thmptoms appear for even slight physical activities but the patient is fine at rest. \\
\hline
\end{tabular}


Table 3. Characteristics of the Study Population $(n=111)$

\begin{tabular}{|c|c|}
\hline Characteristics & \\
\hline Age & $58(47-65)$ \\
\hline BMI & $24.7(21-29.2)$ \\
\hline Men & $72(65 \%)$ \\
\hline NYHA I & $62(55.8 \%)$ \\
\hline NYHA II & $30(27 \%)$ \\
\hline NYHA III & $19(17.1 \%)$ \\
\hline $\operatorname{LVEF}(\leq 50 \%)$ & $54(48.6 \%)$ \\
\hline LVEF $(>50 \%)$ & $57(51.3 \%)$ \\
\hline \multicolumn{2}{|l|}{ Patient history } \\
\hline $\mathrm{AF}$ & $34(31 \%)$ \\
\hline Moderate-to severe CKD $\left(\mathrm{eGFR}<60 \mathrm{~mL} / \mathrm{min} / \mathrm{m}^{2}\right)$ & $47(42 \%)$ \\
\hline Hypertension & $53(48 \%)$ \\
\hline Diabetes & $24(22 \%)$ \\
\hline $\mathrm{ICD} / \mathrm{PM}$ & $17(15 \%)$ \\
\hline \multicolumn{2}{|l|}{ Etiology of heart failure } \\
\hline Ischemic & $49(44.1 \%)$ \\
\hline Valvular & $17(15.3 \%)$ \\
\hline Hypertensive & $22(19.9 \%)$ \\
\hline Idiopathic & $20(18 \%)$ \\
\hline Others & $3(2.7 \%)$ \\
\hline \multicolumn{2}{|l|}{ Biology } \\
\hline eGFR $\left(\mathrm{mL} / \mathrm{min} / \mathrm{m}^{2}\right)$ & $67(54-82)$ \\
\hline Hematocrit (\%) & $42(39-45)$ \\
\hline Capillary BNP (pg/mL) & $400(245-678.75)$ \\
\hline Plasma BNP (pg/mL) & $399(246.5-699)$ \\
\hline Bilirubin $(\mu \mathrm{mol} / \mathrm{L})$ & $13(9-17)$ \\
\hline \multicolumn{2}{|l|}{ Therapy } \\
\hline Beta-blocker & $100(90 \%)$ \\
\hline Diuretics & $82(74 \%)$ \\
\hline ACE-I/ARB & $94(85 \%)$ \\
\hline
\end{tabular}

BMI: body mass index; NYHA: New York Heart Association; LVEF: left ventricular ejection fraction; CKD: chronic kidney disease; eGFR: estimated glomerular filtration rate; ICD: implantable cardioverter defibrillator; PM: pace-maker; BNP: B-type natriuretic peptide; ACE-I: angiotensin converting enzyme inhibitor; ARB: angiotensin receptor blocker.

of detection were $10-5,000 \mathrm{pg} / \mathrm{mL}$. Unlike the Alere ${ }^{\mathrm{TM}}$ Heart Check, the Abbott Architect System required plasma to be obtained from EDTA whole blood by centrifugation at $3,500 \mathrm{~g}$ for $15 \mathrm{~min}$ at $4{ }^{\circ} \mathrm{C}$, within $4 \mathrm{~h}$ after collection. Results were provided within $15 \mathrm{~min}$.

\section{Analytical performance: precision}

Repeatability was assessed by performing six repeated measures of the low and high BNP controls on the same day by the same operator using strips and controls from the same batch. Reproducibility was assessed by performing six measures of the low and high BNP controls per day, over three consecutive days using three different strip batches.

\section{Statistical analysis}

All statistical analyses were performed using the Stata version 10 statistical software (StataCorp LP, College Station, TX, USA). Values were expressed as median (interquartile range), 


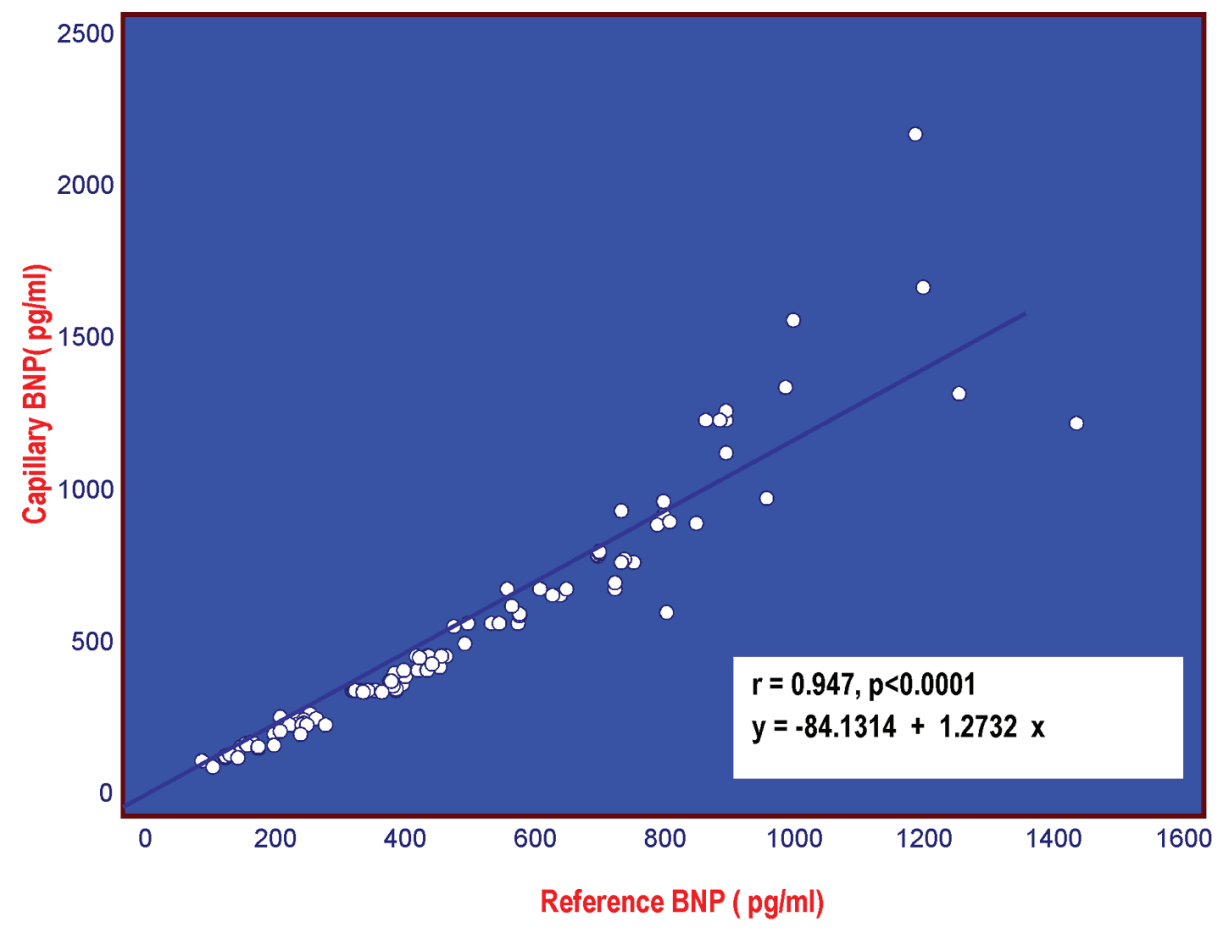

Figure 1. Distribution of capillary BNP measured on the Alere Heart Check system according to plasma BNP measured on the Abbott Architect System among the 111 patients with CHF, examined in the study.

mean \pm SD or number $(\%)$ as appropriate. The difference between capillary and plasma BNP was assessed by Mann-Whitney test (independent samples). Linear regression analysis was made for evaluating the relationship between the BNP values, respectively measured by means of the Alere ${ }^{\mathrm{TM}}$ Heart Check System and Abbott Architect System, the latter being used as reference method. Thus, the regression coefficient (slope) and the correlation coefficient concerning the two methods of BNP measurement were calculated. Bland-Altman plot was built for highlighting the difference between the two techniques for the calculated BNP value, by plotting this difference against the averages of the two techniques. Receiver operator characteristic curves were generated for evaluating the diagnostic accuracy of the two tests (Alere ${ }^{\mathrm{TM}}$ Heart Check and Abbott Architect). A P-value $<0.05$ was considered significant.

\section{Results}

\section{Clinical performance: characteristics of the study popula- tion}

From February 2013 to January 2015, we enrolled 111 patients with stable CHF, mostly men (65\%), whose characteristics are presented in Table 3. Fifty-four patients $(48.6 \%)$ had a reduced ejection fraction $(\leq 50 \%)$ mainly due to ischemic cardiac disease, and 49 (44\%) had symptomatic heart failure (NYHA functional classes II-III). The patients underwent two types of blood sampling, one venipuncture and one fingertip capillary blood sample.

\section{Comparison between Alere ${ }^{\mathrm{TM}}$ Heart Check BNP test and the reference method}

Overall, the BNP values obtained ranged between 95 and 2,178 $\mathrm{pg} / \mathrm{mL}$ using the Alere ${ }^{\mathrm{TM}}$ Heart Check, and between 89 and $1,435 \mathrm{pg} / \mathrm{mL}$ using the Architect System. There was a strong positive correlation between the two measurements $(\mathrm{r}=0.947$, $\mathrm{P}<0.0001$ ), although the values diverged more when BNP was higher than $1,500 \mathrm{pg} / \mathrm{mL}$ (Fig. 1). Bland-Altman analysis confirmed this difference (bias of $46.9 \mathrm{pg} / \mathrm{mL}$ ), some values above $1,500 \mathrm{pg} / \mathrm{mL}$ being outside the $95 \%$ limit of agreement (Fig. 2).

We compared both methods for their diagnostic accuracy in distinguishing the asymptomatic CHF patients, i.e. belonging to NYHA functional class I, from those with symptoms, i.e. belonging to NYHA functional classes II-III. For this purpose, we generated specific receiver operating characteristic curves. The methods (Alere ${ }^{\mathrm{TM}}$ Heart Check vs. Abbott Architect) showed similar diagnostic performances (Figs. 3 and 4) with values of area under the curve (AUC) of 0.983 and 0.984 , respectively, and equivalent sensitivities, specificity, and positive and negative likelihood ratios.

We subsequently compared the results from capillary and plasma BNP measurements, according to the NYHA functional classification of dyspnea (Figs. 5-7). Below there are the pertinent findings, that we have found for each NYHA class by measuring the BNP levels in capillary whole blood (Alere Heart Check System) and EDTA plasma from venous sample (Abbott Architect System) respectively, expressed as median plus interquartile range. There was no statistical difference 


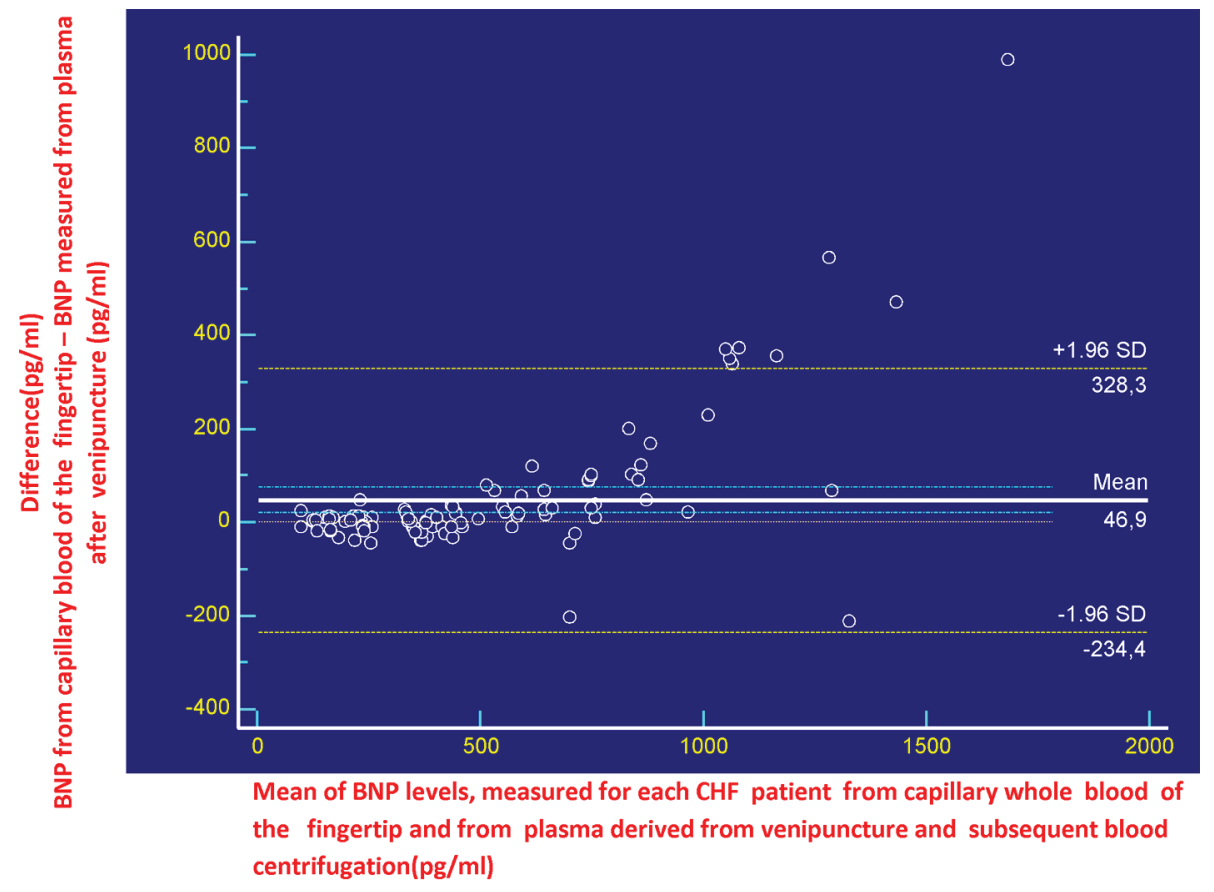

Figure 2. Bland-Altman comparison of capillary and plasma BNP.

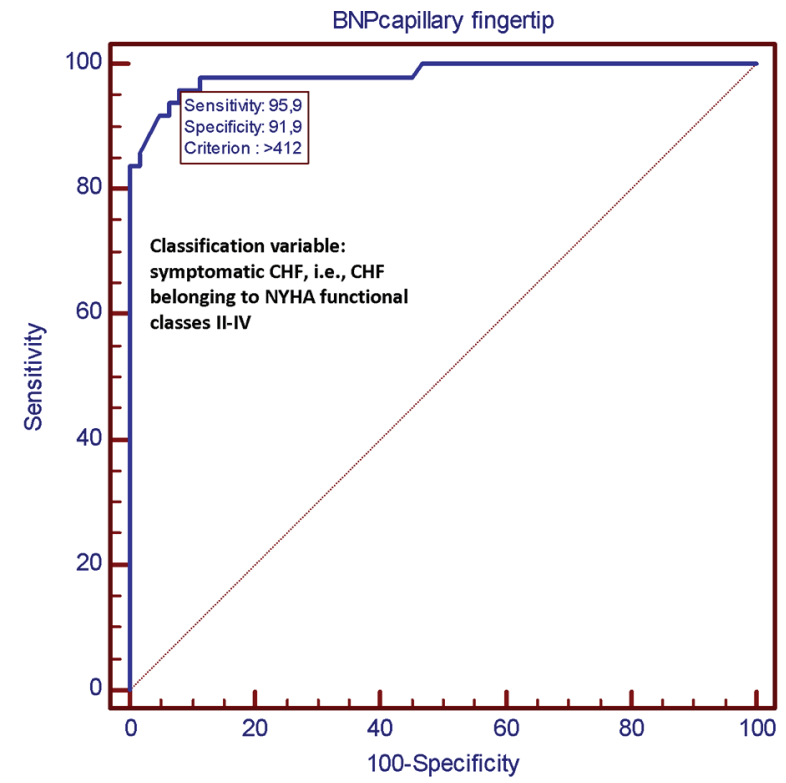

Figure 3. In this ROC plot, there is the representation of the very good diagnostic performance $(A \cup C=0.983)$ of the Alere Check System as a tool for predicting a clinical picture of CHF belonging to NYHA classes II-IV. By adopting this method, the best diagnostic accuracy for identifying a condition of NYHA class II or higher has been attributed to the BNP threshold value of $412 \mathrm{pg} / \mathrm{mL}$. This means that this value, when derived from a measurement made by the Alere Check System on capillary blood, is associated to the presence of heart failure symptoms with a sensitivity of $95.9 \%$ and a specificity of $91.9 \%$ (note on top of the graph). CHF: chronic heart failure; NYHA: New York Heart Association; ROC: receiver operating characteristic; AUC: area under the curve; BNP: B-type natriuretic peptide; pg: picogram.

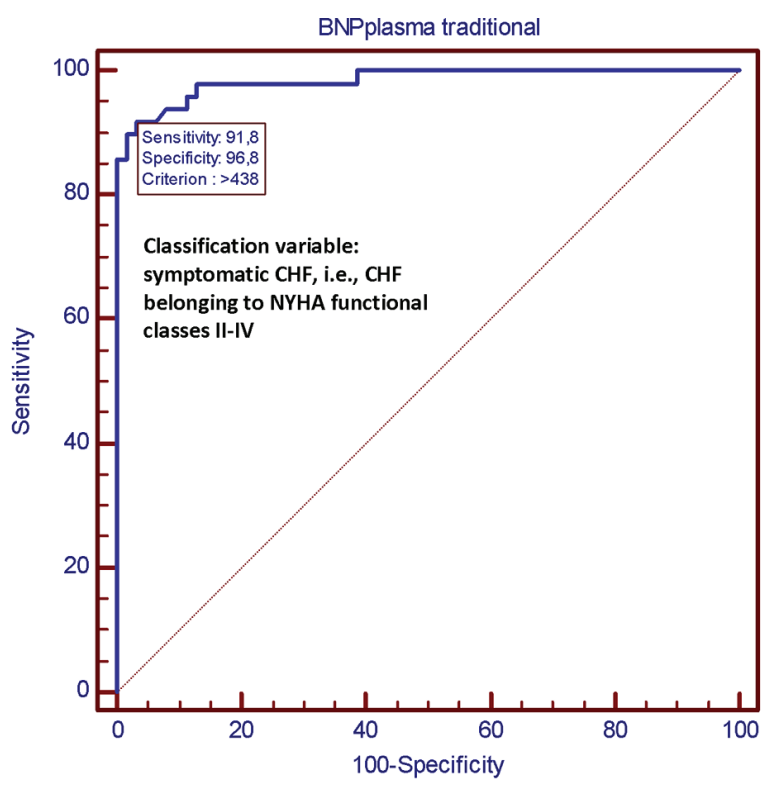

Figure 4. In this ROC plot, there is the representation of the very good diagnostic performance $(A \cup C=0.984)$ of the Abbott Architect System as a tool for predicting a clinical picture of CHF belonging to NYHA classes II-IV. The criterion value of $438 \mathrm{pg} / \mathrm{mL}$ has been identified as the BNP value that exhibits the best diagnostic accuracy for predicting the presence of symptoms of heart failure. This means that this value, when the BNP measurement is done from plasma using the Abbott Architect System, is associated with heart failure symptoms with the best combination of sensitivity and specificity $(91.8 \%$ and $96.8 \%$, respectively). CHF: chronic heart failure; NYHA: New York Heart Association; ROC: receiver operating characteristic; AUC: area under the curve; BNP: B-type natriuretic peptide; pg: picogram. 


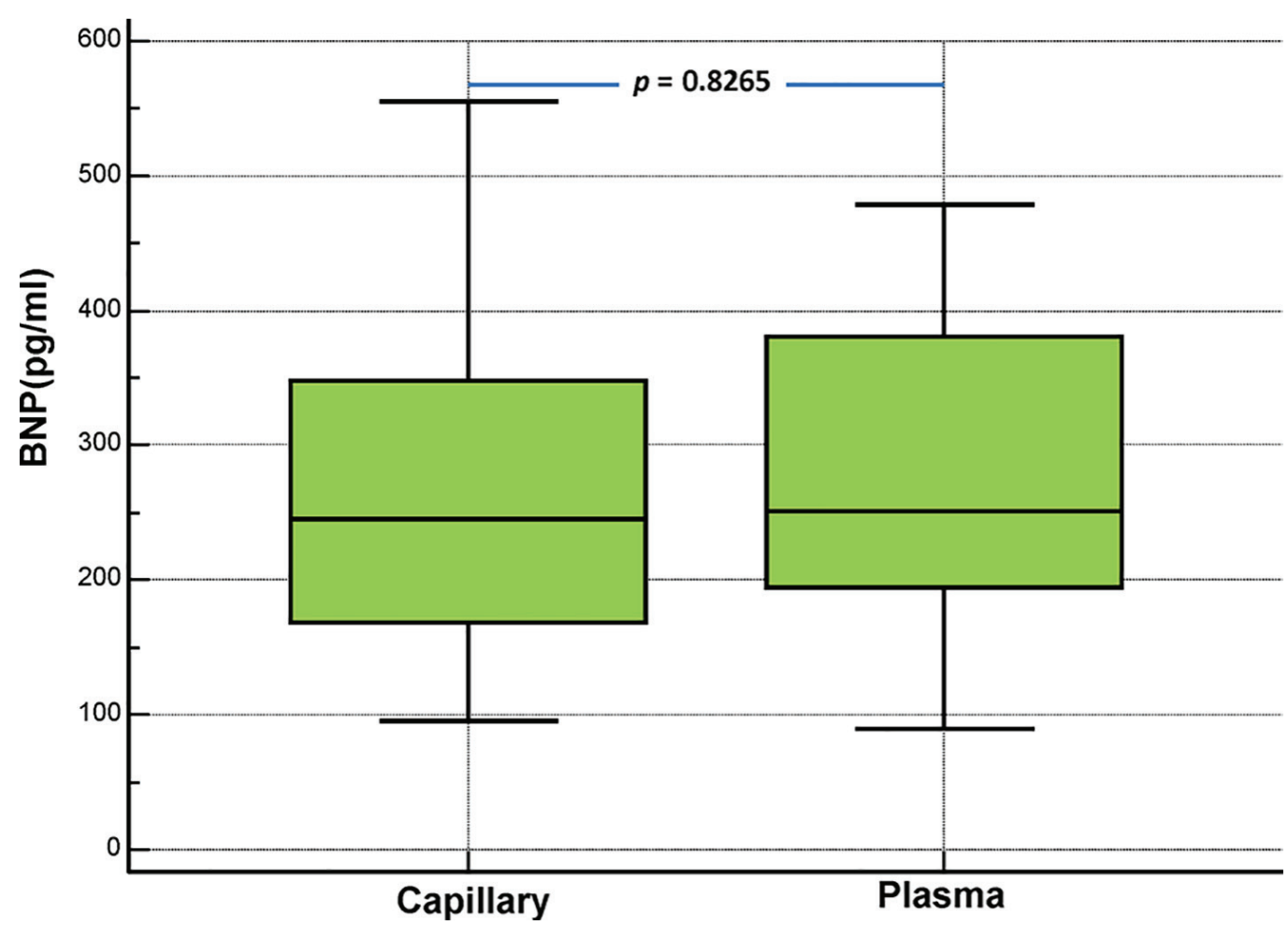

Figure 5. Comparison of capillary (Alere Heart Check System) and plasma (Abbott Architect System) BNP levels in patients with chronic heart failure NYHA class I $(n=62)$.

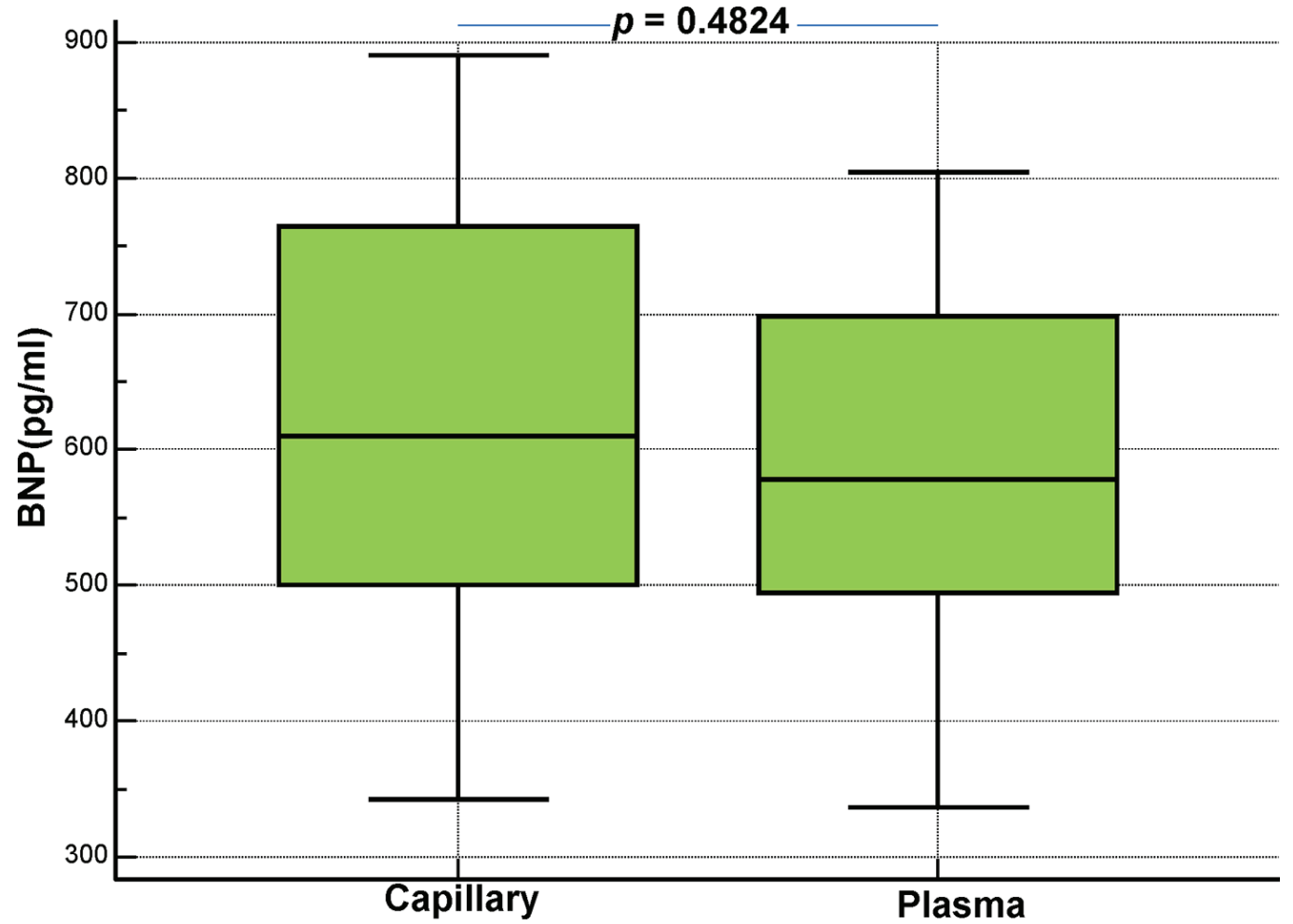

Figure 6. Comparison of capillary (Alere Heart Check System) and plasma (Abbott Architect System) BNP levels in chronic heart failure patients with NYHA class II $(n=30)$. 


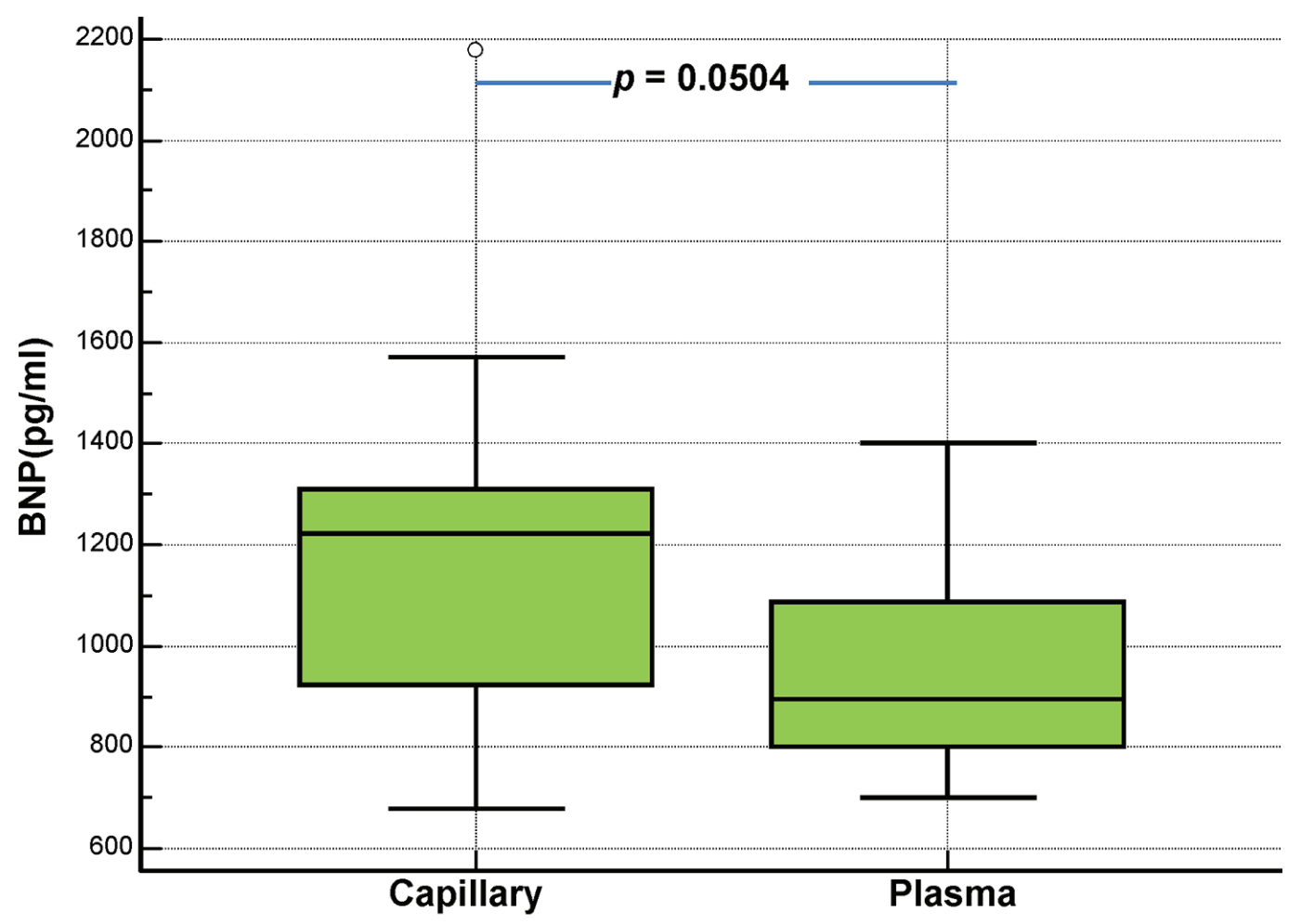

Figure 7. Comparison of capillary (Alere Heart Check System) and plasma (Abbott Architect System) BNP levels in chronic heart failure patients with NYHA class III $(n=19)$.

between capillary and plasma BNP according to the NYHA class of patients (NYHA I: $245 \mathrm{pg} / \mathrm{mL}(168.5$ - 348.25) vs. $251 \mathrm{pg} / \mathrm{mL}$ (194 - 381); NYHA II: 610.5 pg/mL (500 - 765) vs. $578.5 \mathrm{pg} / \mathrm{mL}$ (495 - 699); NYHA III: 1,223 pg/mL (923.75 $1,309.75)$ vs. $897 \mathrm{pg} / \mathrm{mL}(802.5-1,087.5))$.

\section{Discussion}

In this study, we evaluated the clinical performance of a new method for the hematochemical diagnosis of cardiac decompensation at the patient's bedside, the Alere ${ }^{\mathrm{TM}}$ Heart Check system, by comparing it with our standard laboratory method (Abbott Architect), which measures the plasma BNP. Overall, the Alere ${ }^{\mathrm{TM}}$ Heart Check system showed good performance as evidenced by the good correlation with the results of the Abbott Architect found in the entire investigated cohort. Due to technical limitations, the POC testing methods are usually less accurate than the automata-based methods for quantifying biomarkers. The Alere ${ }^{\mathrm{TM}}$ Heart Check system is no exception, and its accuracy falls within the range observed for other POCs measuring BNP at low concentrations of BNP [5] (Table 1). For higher concentrations of BNP, the Alere ${ }^{\mathrm{TM}}$ Heart Check system exhibited a relatively high coefficient of variation (18\%) compared to other POCs, which is in line with the correlation rather low between the values of capillary (Alere ${ }^{\mathrm{TM}}$ Heart Check) and plasma (Abbott Architect) BNP at higher concentrations $(>1,500 \mathrm{pg} / \mathrm{mL})$, as found in NYHA class III (see also the box-and-whisker plots displayed in Fig- ures 5-7). A similar variation, related to the concentration, was also observed in lab-based essays such as the i-STAT Abbott (Fig. 1). These results suggest that the Alere ${ }^{\mathrm{TM}}$ Heart Check system is an optimal tool for accurate identification of BNP levels, when they are located within its low-to-mid range. Overall, these data strongly suggest that the Alere ${ }^{\mathrm{TM}}$ Heart Check system is reliable for measuring BNP in patients with CHF, particularly those within NYHA classes I-III, although important changes to the BNP should be confirmed by lab-based tests. From a practical standpoint, this system is the only test for BNP from capillary blood; the Abbott iSTAT and the Alere ${ }^{\mathrm{TM}}$ Triage systems used in emergency departments measure BNP using either venipuncture EDTA whole blood (Abbott i-STAT) or EDTA plasma (Alere ${ }^{\mathrm{TM}}$ Triage). Therefore, the Alere ${ }^{\mathrm{TM}}$ Heart Check is the only non-invasive system that uses capillary blood from the fingertip and could easily be implemented as a rapid method for evaluating BNP by the general practitioner, the community cardiologist or for home monitoring, as evidenced by the recent HABIT trial [10]. The differences observed between the two methods (Alere $^{\mathrm{TM}}$ Heart Check and Abbott Architect) are quite small, which suggests that both could be used, in alternation, to follow the efficacy of treatment in patients with CHF. Moreover, some ergonomic improvements could be made to facilitate the deposition of the blood droplet on the strip, which would result in fewer errors. In addition, the machine lacks traceability of the results since patients' ID cannot be entered into the machine. Importantly, the data we obtained showed the Alere ${ }^{\mathrm{TM}}$ Heart Check BNP assay performed $(\mathrm{AUC}=0.983)$ as well 
as the Abbott Architect assay (AUC = 0.984) in distinguishing patients with asymptomatic (NYHA I) and symptomatic (NYHA II-III) CHF. These results suggest that the Alere ${ }^{\mathrm{TM}}$ Heart Check BNP assay could be used in an emergency to triage dyspneic patients. However, our data suggest that more studies are needed to validate this method in emergency conditions. Indeed, capillary (Alere ${ }^{\mathrm{TM}}$ Heart Check) BNP was biased by $46.9 \mathrm{pg} / \mathrm{mL}$ (Bland-Altman plot in Fig. 2) compared to plasma (Abbott Architect) BNP. Currently, the algorithm available for the diagnosis of acute decompensated heart failure (ADHF) in international guidelines refers to a BNP cutoff of $100 \mathrm{pg} / \mathrm{mL}$, i.e. ADHF can be excluded in acutely dyspneic patients whose BNP levels fall below this threshold value $[1,2]$. Given the bias observed in our Bland-Altman comparison, the use of the Alere ${ }^{\mathrm{TM}}$ Heart Check system in emergency conditions will most likely overestimate patients who would fall into the gray zone of the BNP (100 - $400 \mathrm{pg}$ / $\mathrm{mL}$ ), among acutely dyspneic patients, unless a new threshold is determined for this system.

Our study has many limitations. First, it was a study based on only two participating centers, with a small population younger than the current population with $\mathrm{CHF}$ and a few women. However, the range of BNP measured was sufficiently broad to test the limits of the measurements. Compared to other POCs available for blood glucose, INR or plasma BNP, we postulate a higher cost than laboratory-based methods. However, cost-effectiveness should be evaluated in a larger-scale study, according to the aimed application, e.g. remote vs. inhospital monitoring.

\section{Conclusions}

The Alere ${ }^{\mathrm{TM}}$ Heart Check BNP test is a good POC for the management of heart failure despite a relatively poor precision in higher BNP values. There was good agreement between the Alere $^{\mathrm{TM}}$ Heart Check system and the lab-based test (Abbott Architect System). Thus, further studies are required to evaluate the real cost and real application of this device in emergency departments or by patients at home.

\section{Financial Disclosures}

There are no financial disclosures to be reported for the present article and the research work that is described in it.

\section{References}

1. McMurray JJ, Adamopoulos S, Anker SD, Auricchio A, Bohm M, Dickstein K, Falk V, et al. ESC Guidelines for the diagnosis and treatment of acute and chronic heart failure 2012: The Task Force for the Diagnosis and Treatment of Acute and Chronic Heart Failure 2012 of the European Society of Cardiology. Developed in collaboration with the Heart Failure Association (HFA) of the ESC. Eur Heart J. 2012;33(14):1787-1847.

2. Yancy CW, Jessup M, Bozkurt B, Butler J, Casey DE, Jr., Drazner MH, Fonarow GC, et al. 2013 ACCF/AHA guideline for the management of heart failure: a report of the American College of Cardiology Foundation/American Heart Association Task Force on Practice Guidelines. J Am Coll Cardiol. 2013;62(16):e147-239.

3. Cheng V, Kazanagra R, Garcia A, Lenert L, Krishnaswamy P, Gardetto N, Clopton P, et al. A rapid bedside test for B-type peptide predicts treatment outcomes in patients admitted for decompensated heart failure: a pilot study. J Am Coll Cardiol. 2001;37(2):386-391.

4. Vogeser M, Jacob K. B-type natriuretic peptide (BNP) - validation of an immediate response assay. Clin Lab. 2001;47(1-2):29-33.

5. Ro R, Thode HC, Jr., Taylor M, Gulla J, Tetrault E, Singer AJ. Comparison of the diagnostic characteristics of two B-type natriuretic peptide point-of-care devices. J Emerg Med. 2011;41(6):661-667.

6. Shah K, Terracciano GJ, Jiang K, Maisel AS, Fitzgerald RL. Comparability of Results between Point-of-Care and Automated Instruments to Measure B-type Natriuretic Peptide. West J Emerg Med. 2010;11(1):44-48.

7. Heartcheck BNP test strip package insert 0017 spec-0363 rev. 1 2010/09, Alere Technologies Ltd, Stirling, Scotland.

8. Lang NN, Wong CM, Dalzell JR, Jansz S, Leslie SJ, Gardner RS. The ease of use and reproducibility of the Alere Heart Check System: a comparison of patient and healthcare professional measurement of BNP. Biomark Med. 2014;8(6):791-796.

9. Triage $\AA$ BNP test product insert, 2011, Alere Technologies Ltd., Stirling, Scotland.

10. Maisel A, Barnard D, Jaski B, Frivold G, Marais J, Azer M, Miyamoto MI, et al. Primary results of the HABIT Trial (heart failure assessment with BNP in the home). J Am Coll Cardiol. 2013;61(16):1726-1735. 